\title{
EFFECT OF A SHARP BEND ON THE TRANSMISSION OF NEUTRON GUIDE TUBES OF CIRCULAR CROSS SECTION
}

\author{
WALDEMAR MAYSENHÖLDER \\ School of Mathematical and Physical Sciences, University of Sussex, Brighton, Sussex, England
}

Received 24 June 1976

A Monte Carlo calculation has been performed to investigate the effect of a sharp bend on the transmission of a neutron guide tube for neutrons in the velocity range of cold to ultra-cold. The results are compared with the predictions of the analytical solution to a simple related case in two dimensions.

\section{Introduction}

The calculations described below were stimulated by experiments with a neutron guide tube consisting of several segments of straight copper pipe of circular cross section. Neutron guide tubes have been studied by Maier-Leibnitz and Springer ${ }^{1}$ ) (rectangular cross section, continuously curved), Schaerpf and Eichler ${ }^{2}$ ) and Steyerl $^{3}$ ) (circular cross section, continuously curved pipes and polygonal arrangements of straight sections) and Brown et al. ${ }^{4}$ ) (Monte Carlo calculation for straight pipes). However, no detailed investigation has been published of the effect of a single abrupt bend on the transmission probability for neutrons in the velocity range of cold and ultra-cold neutrons. As this problem seems too complicated to be solved analytically, a Monte Carlo calculation of the effect has been performed.

\section{A simple related case with an analytic solution}

To get an idea of the outcome of the computations, consider the 2-dimensional case in fig. 1a. Suppose neutrons with velocity $v$ enter the "channel" along the $z$-axis. In velocity space (fig. $1 b$ ) they are represented by points (uniformly) distributed on the circumference of the semicircle with radius $v=\left(v_{y}^{2}+v_{z}^{2}\right)^{\frac{1}{2}}$. The condition for total reflection at the boundaries $y= \pm R$ is $\left|v_{y}\right| \leqslant v_{\mathrm{c}}$, where $v_{\mathrm{c}}$ is the critical velocity. [ $v_{\mathrm{c}}$ can be calculated from the index of refraction and depends on the material of the surface $\left.{ }^{5}\right)$ ]. Thus, only particles between $\mathrm{A}$ and $\mathrm{B}$ on the circumference are reflected. After the bend, characterized by the angle $\lambda$, the condition for total reflection is similarly $\left|v_{y^{\prime}}\right| \leqslant v_{\mathrm{c}}$. Therefore, only particles between $\mathrm{C}$ and $\mathrm{B}$ satisfy the conditions for transmission through the bent "channel".

The ratio $R_{\mathrm{s}}$ of the arcs $\widehat{\mathrm{CB}}$ and $\widehat{\mathrm{AB}}$, which can be regarded as the number of reflected neutrons after the bend divided by the number of reflected neutrons before the bend, is a measure of the effect of the bend. $R_{\mathrm{s}}$ is evaluated in terms of $\varphi_{\mathrm{c}}$ instead of $v \cdot \varphi_{\mathrm{c}}$ is the critical glancing angle and related to $v$ by $\sin \varphi_{\mathrm{c}}=v_{\mathrm{c}} / v$. Neutrons with an angle $\varphi \leqslant \varphi_{\mathrm{c}}$ with respect to the surface are totally reflected. $R_{\mathrm{s}}\left(\varphi_{\mathrm{c}}\right)$ has three ranges:

$$
\begin{array}{cl}
\text { (I) } 0 \leq \varphi_{\mathrm{c}} \leq \frac{1}{2} \lambda, & R_{\mathrm{s}}\left(\varphi_{\mathrm{c}}\right)=0 ; \\
\text { (II) } \frac{1}{2} \lambda \leq \varphi_{\mathrm{c}} \leq\left(\frac{1}{2} \pi-\frac{1}{2} \lambda\right), & R_{\mathrm{s}}\left(\varphi_{\mathrm{c}}\right)=1-\lambda /\left(2 \varphi_{\mathrm{c}}\right) ; \\
\text { (III) }\left(\frac{1}{2} \pi-\frac{1}{2} \lambda\right) \leq \varphi_{\mathrm{c}} \leq \frac{1}{2} \pi, & R_{\mathrm{s}}\left(\varphi_{\mathrm{c}}\right)=2-\pi /\left(2 \varphi_{\mathrm{c}}\right) .
\end{array}
$$

The most essential features of $R_{\mathrm{s}}\left(\varphi_{\mathrm{c}}\right)$ are the cut-off at $\varphi_{\mathrm{c}}=\frac{1}{2} \lambda$ and that $R_{\mathrm{s}}(\lambda)=\frac{1}{2}$. We shall compare these with the results of the Monte Carlo calculations for circular tubes later. [Three-dimensional models are far too complicated to solve analytically and some approximations which have been tried lead to a very similar behaviour of $R_{\mathrm{s}}\left(\varphi_{\mathrm{c}}\right)$.]

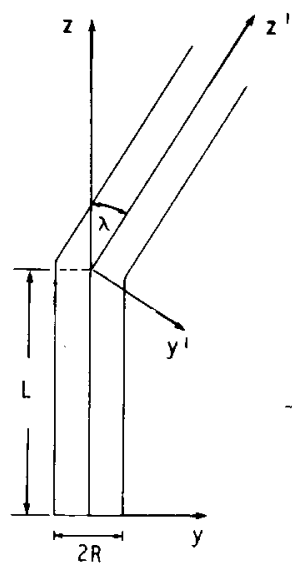

(a)

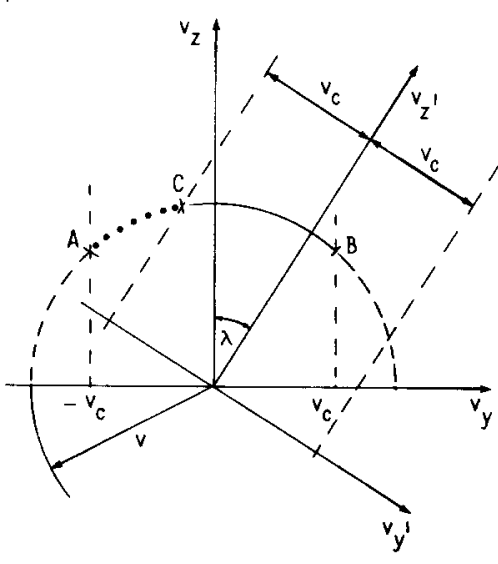

(b)
Fig. 1. (a) Section through bent neutron guide tube in the plane of the bend. (b) Conditions on the neutron velocity for total reffection in the 2 -dimensional velocity space. 


\section{Method}

To simplify the Monte Carlo calculations the following assumptions have been made:

a) Perfect guide tube (no surface roughness etc.).

b) $100 \%$ specular reflection of neutrons with $\varphi \leqslant \varphi_{c}$ and no reflection for neutrons with $\varphi>\varphi_{\mathrm{c}}$.

c) Isotropic distribution of neutrons at the beginning of the pipe.

d) Allowance for reflection losses is made by an attenuation factor $\exp (-\beta N)$, where $N$ is the number of bounces and $\beta$ some attenuation coefficient taken to be independent of angle and velocity.

The calculations are carried out for copper pipes $\left(v_{\mathrm{c}}=5.7 \mathrm{~m} / \mathrm{s}\right)$ with radius $R=5 \mathrm{~cm}$ and a length $L=200 \mathrm{~cm}$ from the beginning to the bend. The angle of the bend is denoted by $\lambda$ as in fig. 1 a.

A particle starts from the surface at the beginning of the pipe and the glancing angle $\varphi_{1}$ remains the same as long as the pipe is straight. After the bend it will have a different angle $\varphi_{2}$ in general. $\varphi$ is defined to be the maximum of $\varphi_{1}$ and $\varphi_{2}$, i.e. $\varphi=\varphi_{2}^{1}$ if $\varphi_{1} \gtrless \varphi_{2}^{2}$. Now the range of these $\varphi^{\prime}$ 's, $0^{\circ}-90^{\circ}$, has been subdivided into 18 sections $\left(0^{\circ}-5^{\circ}, 5^{\circ}-10^{\circ}\right.$, etc. $)$. Counting the particles in their appropriate sections (followed by a normalization) gives the probability densities $p_{1}\left(\varphi_{1}\right)$, $p_{2}\left(\varphi_{2}\right), p(\varphi)$ for a path having the angles $\varphi_{1}, \varphi_{2}, \varphi$ respectively. (The histograms obtained from the computation have been extrapolated to smooth curves as shown in the diagrams.) If now $\varphi_{\mathrm{c}}$ is greater than $\varphi$, a neutron following a particular path is transmitted. The probability of transmission $T\left(\varphi_{\mathrm{c}}\right)$ for a neutron with critical glancing angle $\varphi_{\mathrm{c}}$ is simply

$T\left(\varphi_{c}\right)=\int_{0}^{\varphi_{c}} p(\varphi) \mathrm{d} \varphi$.

For a straight pipe accordingly

$T_{1}\left(\varphi_{\mathrm{c}}\right)=\int_{0}^{\varphi_{\mathcal{c}}} p_{1}\left(\varphi_{1}\right) \mathrm{d} \varphi_{1}$.

\section{Isotropic distribution and effect of a straight pipe}

If a plane is placed in an isotropic distribution, the probability of an angle $\varphi$ with respect to the surface is proportional to $\sin 2 \varphi$. Thus we may consider points on the inner surface at the beginning of the pipe as sources of particles, which have this $\sin 2 \varphi$ angulardistribution. This is simulated by 10310 particles emitted from one point. It turns out that a single point source at $z=0$ may be regarded as approximately representative of an initial isotropic distribution, provided $L / R$ is large enough. For the results change by less than $1 \%$ for a variation of both the position on the circumference and the length from $200 \mathrm{~cm}$ to $150 \mathrm{~cm}$, which is comparable to the statistical error $\leq 0.5 \%$ for $T\left(\varphi_{\mathrm{c}}\right)$ and $T_{1}\left(\varphi_{\mathrm{c}}\right)$. By making use of this fact the Monte Carlo calculation became more economic of time.

The angular distribution in a straight pipe remains constant along the length of the pipe, if there are no reflection losses. Of course, in reality such losses will occur. The simplest way to take account of these is by finally counting a particle with weight $\exp (-\beta N)$, the attenuation factor mentioned above, instead of with unit weight. Fig. 2 shows the collimating effect on the angular distribution to smaller angles with increasing $\beta$. The main purpose of this procedure is to eliminate extreme narrow helical paths with many thousands of reflections per meter, i.e. to make the distribution of neutrons just before the bend more realistic.

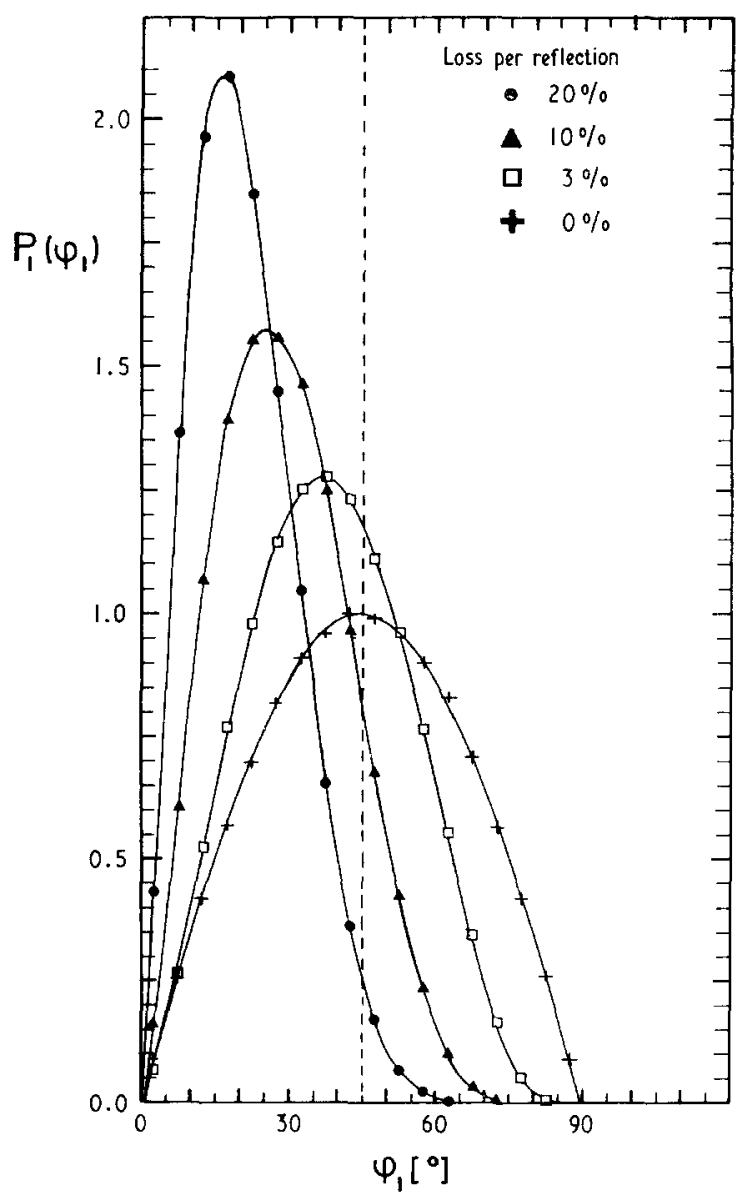

Fig. 2. Angular distribution $P_{1}\left(\varphi_{1}\right)$ at the end of a straight pipe ( $L=200 \mathrm{~cm}, R=5 \mathrm{~cm}$ ) for several values of loss per reflection. This illustrates the collimating effect. 


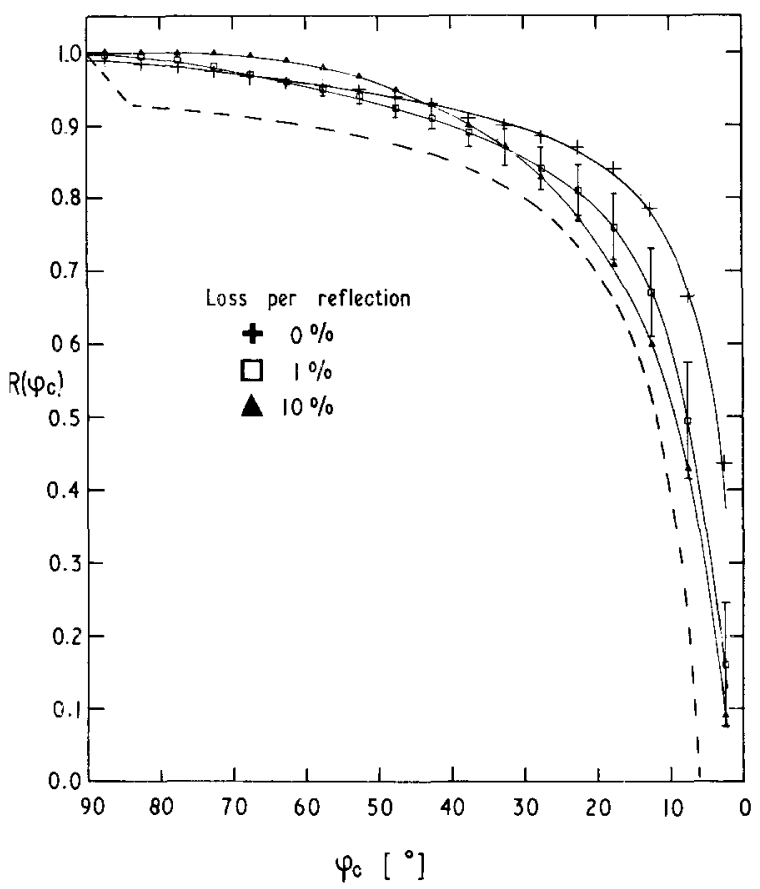

Fig. 3. $R\left(\varphi_{\mathrm{c}}\right)$ at $\lambda=12^{\circ}$ for several values of the loss per reflection. The dashed line represents $R_{\mathrm{s}}\left(\varphi_{\mathrm{c}}\right)$ at $\lambda=12^{\circ}$. The error bars shown on the $1 \%$ loss curve do not vary significantly with loss coefficient.

\section{Results and conclusions}

The effect of the bend can be characterized by the ratio

$R\left(\varphi_{\mathrm{c}}\right)=T\left(\varphi_{\mathrm{c}}\right) / T_{1}\left(\varphi_{\mathrm{c}}\right)$

Unfortunately, $R\left(\varphi_{\mathrm{c}}\right)$ is not independent of $\beta$ and $L / R$. If the calculations were very sensitive to $\beta$, they would be of little value, because the attenuation law is too simple. In fig. $3, R\left(\varphi_{\mathrm{c}}\right)$ is drawn for different values of $\beta$ with $\lambda=12^{\circ}$. The curves with $1 \%$ and $10 \%$ loss per reflection lie rather close together. [For comparison - the statistical error on $R\left(\varphi_{\mathrm{c}}\right)$ decreases nonlinearly from about $8 \%$ for the lowest values of $R\left(\varphi_{\mathrm{c}}\right)$ to $0 \%$ for $R\left(\varphi_{\mathrm{c}}\right)$ approaching unity.] This justifies the assumption that the calculations provide a good estimate of the effect of the bend in spite of the simple attenuation law, at least for small values of $\lambda$. (For $\lambda=90^{\circ}$ the greatest differences between the curves with $1 \%$ and $10 \%$ loss per reflection are about 0.2 .) Fig. 4 shows $R\left(\varphi_{\mathrm{c}}\right)$ for larger values of $\lambda$. The loss per reflection is chosen to be $5 \%$, which is certainly attainable for polished copper pipes. (Ref. 5 quotes an average reflectivity of $97 \%$ or $98 \%$.) $R\left(\varphi_{\mathrm{c}}\right)$ does not

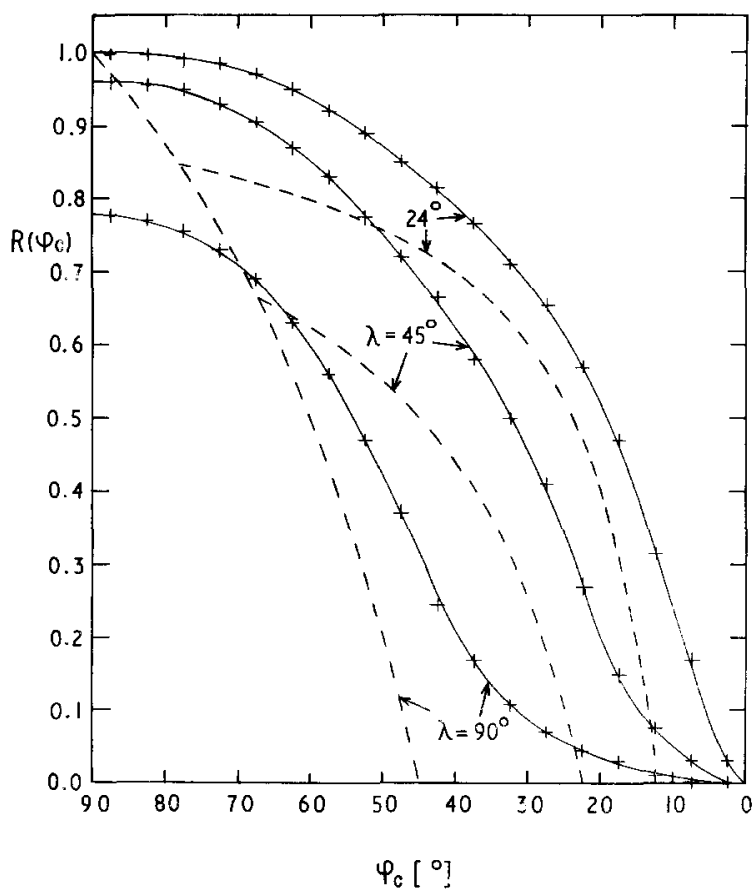

Fig. 4. $R\left(\varphi_{\mathrm{c}}\right)$ (unbroken lines) and $R_{\mathrm{s}}\left(\varphi_{\mathrm{c}}\right)$ (dashed lines) at $\lambda=24^{\circ}, 45^{\circ}$ and $90^{\circ}$ for a loss per reflection of $5 \%$.

reach unity in this diagram. This is due to reflection backwards into the first section of the pipe.

The dotted lines in figs. 3 and 4 are the values of $R_{\mathrm{s}}\left(\varphi_{\mathrm{c}}\right)$ obtained from the simple analytical case. A comparison emphasizes the following points

1) Although the values of $R\left(\varphi_{\mathrm{c}}\right)$ are remarkably reduced, there is no sharp cut-off at $\varphi_{\mathrm{c}}=\frac{1}{2} \lambda$ as as in the 2-dimensional case. Rather $R\left(\frac{1}{2} \lambda\right)$ is of the order of 0.3 .

2) For lower values of $\varphi_{\mathrm{c}}, R\left(\varphi_{\mathrm{c}}\right)$ is always larger than estimated by $R_{\mathrm{s}}\left(\varphi_{\mathrm{c}}\right)$; in particular $R(\lambda)>0.5$ (of the order of 0.6 for $\lambda=12^{\circ}$ and $24^{\circ}$ ). Both these results are probably due to the ease with which particles can get round the corner by means of helical paths, which are possible because of the circular cross section of the guide tube.

3) The simple analytical value $R_{\mathrm{s}}\left(90^{\circ}\right)$ is always unity in contrast to the calculated values of $R\left(90^{\circ}\right)$ shown in fig. 4 . This means that the simple analytical formula cannot describe the loss due to reflection backwards, which becomes important for larger values of $\lambda$.

I am very grateful to Dr. J. M. Pendlebury for advice on this work and for reading the manuscript and to 
Dr. J. Byrne for additional discussions and refinements.

\section{References}

1) H. Maier-Leibnitz and T. Springer, J. Nucl. Energy, Part
A/B, 17 (1963) 217.

2) O. Schaerpf and D. Eichler, J. Phys. E, Sci. Instr. 6 (1973) 774.

3) A. Steyerl, Nucl. Instr. and Meth. 101 (1972) 295.

${ }^{4)}$ M. Brown, R. Golub and J. M. Pendlebury, Vacuum 25 (1975) 61.

5) D. J. Hughes and M. T. Burgy, Phys. Rev. 81 (1951) 498. 